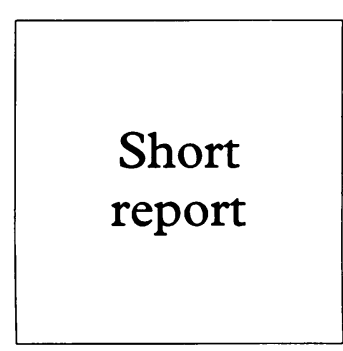

\title{
Metronidazole resistant trichomoniasis successfully treated with paromomycin
}

\author{
D D Coelho
}

Management of a 42 year old female patient diagnosed with trichomoniasis is described. She failed to respond to recommended oral and high dose oral and topical metronidazole. Various options used in previously reported cases of metronidazole resistant trichomoniasis also failed to cure her condition. MIC showed the organism to be resistant to metronidazole. Cure was achieved with the use of topical intravaginal paromomycin.

(Genitourin Med 1997;73:397-398)

Keywords: Trichomonas vaginalis; metronidazole; paromomycin

\section{Introduction}

Trichomonas vaginalis is a sexually transmissible protozoon organism producing a vaginal discharge in women. The standard recommended treatment for over 30 years has been oral metronidazole. Concurrent treatment of sexual partners is recommended to prevent reinfection. Occasionally, cases are reported of trichomoniasis resistant to standard doses of metronidazole. ${ }^{1-4}$ Resolution may occur with higher doses of metronidazole. True resistance to the drug and non-response to metronidazole in tolerable doses have also been reported. A case of trichomoniasis failing to respond to metronidazole is reported below. Successful treatment with the antibiotic paromomycin is described.

\section{Case report}

A 42 year old female patient presented in December 1992 with a 10 month history of persistent vaginal discharge for which she had received intermittent courses of erythromycin. She was found to have trichomoniasis and was prescribed metronidazole $400 \mathrm{mg}$ twice daily for 7 days. She denied any further sexual activity. Her former sexual partner was also treated with metronidazole. $T$ vaginalis was repeatedly isolated on follow up culture over the next few weeks despite further courses of both oral and topical intravaginal metronidazole. The highest oral dose of metronidazole used was $800 \mathrm{mg}$ three times daily for 7 days with $1 \mathrm{~g}$ metronidazole intravaginally nightly. Over the next few months, she was treated without success with oral tinidazole, and nimorazole,

Minimum inhibitory concentration of test organisms and control

Department of $\mathbf{G} \mathbf{U}$ Medicine, St John's Hospital, Wood Street, Chelmsford, Essex, CM2 9BG

D D Coelho

Accepted for publication 25 April 1997

\begin{tabular}{lr}
\hline Control & \multicolumn{1}{c}{ Test } \\
\hline 20 December $1994:$ & \\
Aerobic $2.0 \mathrm{mg} / \mathrm{ml}$ & $125.0 \mathrm{mg} / \mathrm{ml}$ \\
Anaerobic $1.0 \mathrm{mg} / \mathrm{ml}$ & $2.0 \mathrm{mg} / \mathrm{ml}$ \\
October $1995:$ & \\
Aerobic $2.0 \mathrm{mg} / \mathrm{ml}$ & $64.0 \mathrm{mg} / \mathrm{ml}$ \\
Anaerobic $1.0 \mathrm{mg} / \mathrm{ml}$ & $2.0 \mathrm{mg} / \mathrm{ml}$ \\
\hline
\end{tabular}

mebendazole, and intravaginal clotrimazole, providone-iodine, Aci-Jel, nonoxynol-9 pessaries, and hydrogen peroxide douches. This was followed by a full course of inactivated lactobacilli vaccines (Solotrichovac) followed by a booster 1 year later.

She continued to be symptomatic and $T$ vaginalis was isolated repeatedly throughout this period. She had a hysterectomy undertaken in 1993 for menorrhagia. Initial minimum inhibitory concentration (MIC) of metronidazole for the organism isolated is shown in the table. The organism was resistant to metronidazole. A 6 month metronidazole free period was tried in the hope of reversion of the organisms to susceptibility. A repeat MIC study showed persistent resistance and a rechallenge with metronidazole failed to achieve a cure. Hospitalisation for a trial of metronidazole was also considered. It was felt, however, that there was little advantage to be gained as metronidazole is well absorbed when given orally and high intravaginal levels were already being achieved with intravaginal metronidazole. Paromomycin had been obtained to treat an HIV positive patient with intractable Cryptosporidium positive diarrhoea. The accompanying data sheet suggested that the drug was active against $T$ vaginalis. No suitable preparation was available, however, for intravaginal use. The patient agreed to treatment after discussion. A preparation was made by the hospital pharmacy consisting of paromomycin suspended in Unguentum Merck which could be introduced into the vagina using a vaginal applicator filled via a syringe. She was prescribed one applicator full consisting of $250 \mathrm{mg}$ of paromomycin per applicator, daily for 7 days. The patient discontinued treatment after 5 days because of external genital soreness. Examination revealed tender areas of erosion on the left vulva. Apart from a small amount of creamy fluid in the vaginal vault, she had no evidence of vaginitis. Microscopy and culture for $T$ vaginalis were reported negative. No organism was isolated from a swab taken from the 
eroded areas. The vulval lesions settled rapidly with saline soaks. On follow up, she had no clinical vaginitis and a series of four negative cultures for $T$ vaginalis were obtained over a 6 week period.

\section{Discussion}

Paromomycin is a mixture of microbial substances produced by strains of Streptomyces rimosus var paromomycinus. ${ }^{5}$ It is poorly absorbed from the gastrointestinal tract. It has been used in the treatment of intestinal amoeba and tapeworm infections and more recently in cryptosporidiosis in AIDS patients. It had been shown to treat trichomoniasis with cure achieved in $85 \%$ of patients when administered topically as a vaginal pessary. ${ }^{6} \mathrm{Re}$ infection from an untreated male partner was assumed to be the cause of treatment failure. The dose of drug used in the initial report was $25 \mathrm{mg}$ daily for 20 days. This compares with the dose of $250 \mathrm{mg}$ daily for 5 days used in this patient. A higher dose of paromomycin was elected in this patient to avoid possibly inducing resistance to the drug. The erosion seen was presumed to be caused by the drug preparation, and felt to be probably dose related. Inflamation has been reported with the topical use of paromomycin in combination with methylbenzethonium chloride in treatment of cutaneous leishmaniasis. ${ }^{\text {? }}$

Hopefully, further studies of its use in vaginal trichomoniasis should clarify this. The successful use in this case and in a recently reported case ${ }^{8}$ suggests that this drug could be a useful reserve for patients infected with metronidazole resistant $T$ vaginalis. Since the introduction of metronidazole over 30 years ago, successful treatment of $T$ vaginalis has been almost universally reliant on use of this drug despite reports of successful use of alternative preparations. ${ }^{9}$ Some proved topical treatments are no longer available. Many of these regimens failed to result in cure in this patient. Controlled trials with paromomycin, re-examining various doses and duration of treatment should be undertaken. The question still remains, however, of how partners of patients with resistant $T$ vaginalis are to be treated if reinfection is to be avoided.

MIC studies were undertaken at the Public Health Laboratory, Newcastle-upon-Tyne.

1 Dombrowski MP, Sokol RJ, Brown WJ, Bronsteen RA. Intravenous therapy of metronidazole resistan Trichomonas vaginalis. Obstet Gynecol 1987;69:324-5.

2 Grossman JH III, Galask RP. Persistent vaginitis caused by metronidazole resitant trichomonas. Obstet Gynecol 1990, 76:521-2.

3 Ahmed-Jushuf IH, Murray AE, Mckeown J. Managing trichomonal vaginitis refractory to conventional treatmen with metronidazole. Genitourin Med 1988;64:25-9.

4 Sprott MS, Kearns AM, Pattman RS. Trichomonal vaginitis refractory to treatment. Genitourin Med 1988;64 369-72.

5 Reynolds JEF. Martindale: the extra pharmacopoeia. 30th ed. London: Pharmaceutical Press 1993:190-1.

6 Dumont M, Croizat B, Douillet P. Traitement des vaginites a trichomonas par la paromomycine. Gynecol Prat 1964;15:247-51.

7 El-On J, Livshin R, Even-Paz Z, Weinlauch L. Topical treatment of cutaneous leishmanasis. $B M F$ 1985;291: treatment

8 Nyirjesy P, Weitz MV, Grelone SP, Fekete T. Paromomycin for metronidazole resistant trichomonosis. Lancet 1995;346:1110.

9 Watson PG, Pattman RS. Arsenical pessaries in the successful elimination of metronidazole resistant trichomonas vaginalis. Int $\mathcal{F}$ STD AIDS 1996;7:296-7. 